\title{
Editorial Letter
}

\section{Promoting Predictive, Preventive and Personalised Medicine in global scale}

\author{
Olga Golubnitschaja
}

Published online: 10 November 2010

(C) European Association for Predictive, Preventive and Personalised Medicine 2010

\section{Dear EPMA-members \\ Dear Readers of the EPMA-Journal \\ Dear Friends around the world}

2010 - The 1st year of the EPMA-Journal is almost over now. During this year, the EPMA-Journal has been very supportive in announcing the global message of the European Association for Predictive, Preventive \& Personalised Medicine, namely

$\triangleright$ Clinical observations clearly demonstrate that the same therapeutic approaches applied within the same patient cohort lead to good individual outcomes.

$\triangleright$ "First Do No Harm" is the highest imperative of personalised medicine that is credible and feasible only in the case of the application of individualised treatment algorithms.

$\triangleright$ The paradigm change from late interventional approaches to predictive diagnostics followed by a targeted prevention before the pathology manifests itself - is the cost effective concept of advanced healthcare.

$\triangleright$ Predictive perinatal / postnatal diagnostics aimed at the preselection of healthy but disease-predisposed individuals should be followed by effective, individually-created preventive measures - the task for which the highest priority should be given. This measure will lead to a dramatically reduced prevalence of severe chronic pathologies and better long-

O. Golubnitschaja $(\bowtie)$

Department of Radiology,

Rheinische Friedrich-Wilhelms-University of Bonn,

Sigmund-Freud-Str. 25,

53105 Bonn, Germany

e-mail: Olga.Golubnitschaja@ukb.uni-bonn.de

O. Golubnitschaja

European Association for Predictive, Preventive \& Personalised

Medicine,

Brussels, Belgium

URL: www.epmanet.eu term outcomes in the coming generations when treated according to individual parameters and optimally-suited therapeutic modalities.

$\triangleright$ Subsequently, an increased portion of socially active members remaining vibrant, and in excellent physical and mental health, can be expected in the elderly population. This will have the highly desirable effect in helping to solve the accumulating socioeconomical problems of ageing societies around the globe.

$>$ The global challenge of the 21 st century is to make good use of innovative approaches for advanced healthcare that will allow a dramatic increase in the portion of actively contributing members to society. This task requires intelligent political regulations and the creation of new guidelines to advance the healthcare systems that are currently rather resistant to progress in the life sciences.

$>$ Global research and implementation programmes in biomedicine, as well as communication between scientific branches, healthcare-providers, policymakers, educators and organised patient groups plus a consolidation of professional groups will all play a decisive role in driving the overall situation in personalised medicine.

It is evident that in order to reach a maximally positive scenario for the further development in personalised medicine, all the above listed stakeholders should be well consolidated and their interaction well harmonised. How can this objective be realised? Towards this objective, the "European Association for Predictive, Preventive \& Personalised Medicine" is organising the EPMA-World Congress 2011 in Bonn, Germany (September 15-18th) with its specialised Workshops on:

$\triangleright$ Healthcare in overview across the globe

$\triangleright$ Collaboration with global organisations (UNO, WHO, etc.)

$\triangleright$ Granting strategies in PPPM (EU-Commission, NIH, NCI, National Ministries) 

$>$ PPPM-related Education
$\triangle$ Primary Prevention, Body Culture, Sport Medicine
$\triangle$ PPPM in Reproductive Medicine
$\triangle$ PPPM early in life (Neonatology, Paediatrics)
$\triangle$ PPPM in Diabetes care
$\triangle$ PPPM in Neurodegeneration
$\triangle$ PPPM in Cancer
$\triangle$ PPPM in Cardiovascular Pathologies
$>$ Clustered PPPM-Centres, Validation and Standards
$>$ Patients" needs: "Round-table" with GPs and Organised Patients' Groups
$>$ Ethical, Legal and Social Issues (ELSI)
$>$ Computer-assisted patient modelling in PPPM; creation of individualised treatment modalities
$>$ Role of Laboratory Medicine in PPPM
$>$ PPPM-related Bio-Banking
$>$ Drug-diagnostics co-development and commercial- isation strategies in the PPPM era
$>$ Private-public partnerships

The EPMA-World Congress will be inaugurated in 2011 and will take place every two years following the 24-months thematic cycle of the EPMA-Journal. There will be some congress specialities, namely:

1. A strong focus on PPPM-related implementation in medical practice: a specialised Workshop will be dedicated to "Clustered PPPM-Centres" with elaborated standards to be followed by the member-centres that allow a distribution of harmonised medical services at the highest level in Europe and around the globe;

2. The integration of the PPPM-related industry: without exception, each thematic workshop will benefit from the issue-related input by industry and the regulators, including innovative $\mathrm{R}$ \& $\mathrm{D}$ concepts, advanced technologies, PPPM-centred products, etc.

The EPMA-World Congress is an important global event promoting the consolidation and overall PPPM-concept as summarised above. This idea is expressed in the letter of support from the Organisation of United Nations and personally—by Mr. Sergei A. Ordzhonikidze, The ViceSecretary-General of UNO:

Organisation of United Nations places emphasis on advances in medical research, training and care as the "key to realizing the Millennium Development Goals and to advancing public health". Further, UNO values EPMA efforts in contributing to developments in the medical field towards our common goals of improving the healthcare and does not doubt that "EPMA World Congress will serve as a strong platform for medical innovation in support of our shared objectives." The Vice-Secretary General of UNO welcomes "in particular, the focus on collaboration with international organisations, which can serve to link the work of the EPMA with the efforts of the international community for global public health."

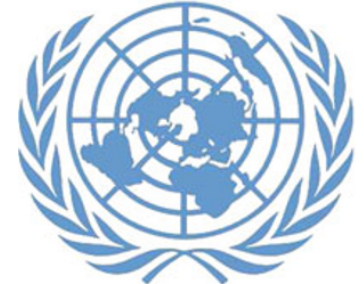

To All of you - A very merry Christmas and a Happy New Year 2011 - the Year, when we meet together for our important mission promoting much progress in the field of Predictive, Preventive \& Personalised Medicine.

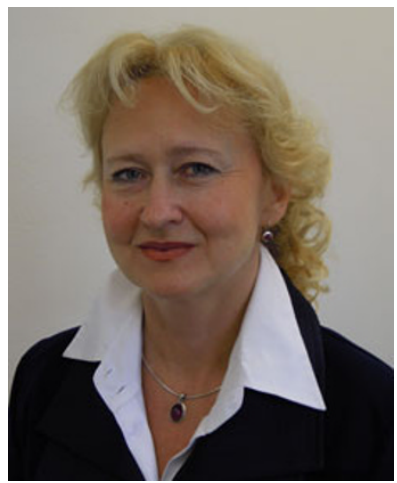

Prof. Dr. Olga Golubnitschaja Editor-in-Chief

Prof. Dr. Olga Golubnitschaja has studied music, journalism, biotechnology, \& medicine in Russia \& Czechoslovakia - all Diplomas with excellent marks / awards. She actively speaks five languages; started to publish since being 12-years old and has been awarded for some literature works. As the professor was / is active at the University in Bratislava, Vienna, Basel, Perugia and Bonn. Since 2000 leads the unit for "Predictive Diagnostics" at the Department of Radiology, University of Bonn and gives courses in European / International Educational Programmes for "Application of Biotechnology in Medicine"; Author of more than 100 international publications in "Predictive \& Personalised Medicine"; External evaluator at the EU-Commission in Brussels and NIH/NCI-grant Commission in Washington D.C, USA. Since 2008 Secretary-General of the "European Association for Predictive, Preventive \& Personalised Medicine" in Brussels; European Representative for "Predictive Diagnostics" at the EDRN / NCI-Network, USA; Reviewer in "Life Sciences" for several European Foundations; Editor of the worldfirst book dedicated to the overall concept of the "Predictive Diagnostics \& Personalised Treatment", Nova Science Publishers, New York, USA, 2009; Co-Editor of "Personalised Medicine", Book-series "Health Academy", Dresden 2010; Scientific activities in Early Molecular Diagnostics \& Predictive Blood-Tests in several cardiovascular, neurodegenerative and tumour pathologies, non-invasive perinatal diagnostics. 